\title{
IMPACT OF PSYCHO-SOCIAL STRESS LEVELS ON CLINICAL PROFILE OF ACUTE CENTRAL SEROUS CHORIORETINOPATHY PATIENTS
}

\author{
Ashok Kumar ${ }^{1}$, Sanjay Kumar Mishra ${ }^{1}$, Vikas Ambiya ${ }^{1}$, Ayon Gupta ${ }^{2}$ \\ ${ }^{1}$ Army College of Medical Sciences \& Base Hospital, Delhi Cantt, New Delhi, India \\ ${ }^{2}$ Centre for Community Medicine, All India Institute of Medical Sciences, New Delhi, India
}

\begin{abstract}
Introduction: To study the impact of the level of psycho-social stress on varied clinical presentations of acute Central serous chorioretinopathy (CSCR) patients.
\end{abstract}

\begin{abstract}
Methods: This prospective observational study included 83 patients with acute central serous chorioretinopathy who presented to our out-patient department. All confirmed cases of acute CSCR were subjected to a questionnaire based on social readjustment rating score which included 43 life events that analyzed if psycho-social stress contributed to their present illness. Patients underwent a comprehensive ocular evaluation including best corrected visual acuity, slit lamp examination of anterior segment, fundus examination using slit lamp with a contact lens or non-contact lens, indirect ophthalmoscopy, digital fundus photography, of Spectral Domain-Optical Coherence Tomogram(SD-OCT) and Fundus FluoresceinAngiography(FFA). The stress scores obtained were statistically analyzed with clinical and angiographic parameters obtained in patients.
\end{abstract}

Result: A total of 83 patients were included in the present study. 72(86.74\%) patients were males,the majority of them presenting with unilateral involvement $(n=70 ; 84.33 \%)$. The prevalence of stress level was mild in 5(6.02\%); moderate in 31(37.34\%) and severe in $47(56.62 \%)$ subjects. Severe level of psycho-social stress was significantly associated with bilateral presentation of serous detachment in posterior pole of fundus (17/47; $p$-value=0.04)as well as multiple angiographic leaks on FFA(19/50; $p$-value=0.03).

Conclusion: Higher levels of psycho-social stress can lead to more severe forms of presentation in acute CSCR with a possible sub-optimal visual recovery. Avoidance of stress and relaxation therapies can help in preventing severe forms of acute CSCR.

Keywords: central serous chorio-retinopathy, psycho-social stress, serous detachment

Cite This Article: KUMAR, Ashok et al. Impact of psycho-social stress levels on clinical profile of acute central serous chorioretinopathy patients. International Journal of Retina, [S.I.], v. 3, n. 2, sep. 2020. ISSN 26148536. Available at: https://www.ijretina.com/index.php/ijretina/article/view/115

${ }^{*}$ Correspondence to: Ashok Kumar Assoc Prof, Army College of Medical Sciences \& Base Hospital, Delhi, New Delhi, India smileashok@rediffmail.com

\section{INTRODUCTION}

Central serous chorioretinopathy (CSCR) is a common retinal disorder characterized by serous detachment of the neuro-sensory retina from the retinal pigment epithelium. It is significantly more prevalent in men than in women. ${ }^{1-2}$ At present, neither the etiology nor the pathophysiology of CSCR is completely understood due to absence of any published literature on same. A definitive range of psychological and psycho-physiological variables have been associated with the increased incidence of CSCR. The exact pathophysiology of CSCR is still not clear, but is known to be mainly related to type " $A$ " personality, anxiety, and stress levels. ${ }^{3-6}$

Choroidal vascular hyperpermeability is one of the main etiology implicated in CSCR and also related to its severity and/or persistent activity. $^{7-8}$ It can have varied presentations ranging from single/multiple neurosensory detachments, fibrinous exudation in subretinal fluid and, in certain cases, with bilateral involvement. Psychosocial stressors has also been implicated in etiology of CSCR patients. ${ }^{9-}$ 10 
A Social Readjustment Rating Score including 43 life events that studied if psycho-social stress contributed to an illness or not was designed by Thomas Holmes and Richard Rahe in 1967.11 Each event had a different "weight" for different stress factors called a Life Change Unit.ncreased chances of him being ill. The scores were totaled up for the events that took place during the previous six months. Many studies have analyzed the association of active CSC with psychological factors but their cumulative impact on clinical profile and various presentations of acute CSCR has not been evaluated.1213 In view of paucity of literature on same, we carried out this study to assess the impact of different grades of psychosocial stress factors on clinical presentation of acute CSCR

\section{METHODS}

A prospective observational study was carried out at a Army College of Medical Sciences \& Base Hospital in Delhi, India from June 2017 to May 2019. The study was conducted after obtaining the necessary approval from the institutional ethical committee, and in accordance with the tenets of the Declaration of Helsinki. All study subjects signed a informed consent for participation.

The various inclusion criteria were: all acute cases of confirmed central serous chorioretinopathy presenting to our centre within 4 weeks from the onset of symptoms, with no history of treatment with drugs, laser or history of recurrent episodes in the past.

The exclusion criteria included: (1) history of intake of oral steroids for any disorder; (2) history of treatment with laser for current episode/other retinal condition; (3) patients with documented recurrence central serous chorioretinopathy; (4) other vitreoretinal disorders concurrent with CSCR currently or in the past; (5) currently on any drugs likely to cause serous detachment of retina.

The diagnosis of CSCR was made on the ophthalmoscopic criterion of serous macular detachment with ring reflex and confirmation of Ink blot or/and smoke stack leak on fundus fluorescein angiography(FFA) with neurosensory detachment and/or pigment epithelial detachment on spectral domain-optical coherence tomogram(SD-OCT).

All confirmed cases of acute central serous chorioretinopathy were evaluated based on social readjustment rating score which included 43 life events that studied if psycho-social stress contributed to their present illness. The more number of life events events the patient added up on this scale, the higher the stress score levels achieved. The scores were totaled up for the events that took place in the previous six months. The stress was graded as Mild; if score was less than 150; Moderate if score was 150-299 and Severe if score was 300-600.

The clinical examination included assessment of the best corrected visual acuity(BCVA) in Snellen, slit lamp examination of anterior segment, fundus examination using slit lamp with a contact lens or non-contact lens, indirect ophthalmoscopy,digital fundus photography, SD-OCT and FFA. Patients were also assessed for unilateral/bilateral involvement; single/multiple leaks on FFA; any fibrinous exudation in serous detachment and corresponding stress scores were recorded. The various clinical, angiographic and tomographic features were studied based on stress level scores.

\section{Statistical analysis}

The numerical variables between any two groups (stress level and clinical/angiographic features) were compared using $\chi^{2}$ (chi-square) using statacorp STATA version 15.1. Test. P-value of $<0.05$ was considered as statistically significant.

\section{RESULTS}

A total of 83 patients based on inclusion and exclusion criteria were included in this study. $72(86.74 \%)$ patients were males with the age of $32.48+6.14$ years at presentation, while $11(13.26 \%)$ females had at presentation the age of $34.27+5.72$ years, no statistical significant difference was noted between two groups. The baseline clinical and demographic features of patients at presentation are listed in Table 1.

Table 1. Demographic \& clinical features of patients(n-83)

\begin{tabular}{ll} 
Clinical characteristics & Values \\
\hline Sex(M/F) & $72(86.74 \%) / 11(13.26 \%)$ \\
\hline Age(M/F) & $32.48 \pm 6.14 / 34.27 \pm 5.72$ \\
\hline Laterality(Unilateral/Bilateral) & $70(84.33 \%) / 13(15.67 \%)$ \\
\hline $\begin{array}{l}\text { Angiography } \\
\text { leaks(Single/Multiple) }\end{array}$ & $63(75.90 \%) / 20(24.1 \%)$ \\
\hline $\begin{array}{l}\text { Fibrinous fluid } \\
\text { (Present/Absent) }\end{array}$ & $9(10.84 \%) / 74(89.16 \%)$ \\
\hline $\begin{array}{l}\text { Prevalence of Stress } \\
\text { Scores(Mild/Moderate/Severe) }\end{array}$ & $5(6.02 \%) / 31(37.34 \%) / 47(56.62 \%)$ \\
\hline \begin{tabular}{l} 
Best corrected visual acuity \\
\hline
\end{tabular} & $(0.2-0.6) \log$ MAR units
\end{tabular}


The BCVA was 0.2-0.6 logMAR units with the majority of patients presenting with unilateral involvement $(n=70 ; 84.33 \%)$ as commonly seen in CSCR. The grading of stress level was mild in 5(6.02\%); moderate in 31(37.34\%) and severe in 47(56.62\%) subjects. The BCVA in Mild stress level patients was comparatively better as compared to severe stress levels but it was not found to be statistically significant $(p$ value $=0.323)$ Table 2.

Table 2. BCVA in logMAR at different stress levels

\begin{tabular}{|c|c|c|c|c|c|c|c|}
\hline Level of stress Score & 0.2 & 0.3 & 0.4 & 0.6 & P-value & 0.6 & P-value \\
\hline Low & 3 & 0 & 1 & 1 & & 1 & \\
\hline Medium & 13 & 14 & 1 & 3 & & 3 & \\
\hline High & 23 & 18 & 3 & 3 & 0.323 & 3 & \\
\hline
\end{tabular}

The severe level of stress was significantly associated with bilateral presentation of serous detachment in posterior pole of fundus(17/47; $p$-value $=0.04$ )as shown in Table 3.

Table 3. Effect of stress on laterality of serous retinal detachment

\begin{tabular}{ccccc} 
Level of stress Score & Unilateral & Bilateral & Total & P-value \\
\hline Low & 5 & 0 & 5 & 0.6 \\
Medium & 28 & 3 & 31 & 0.08 \\
High & 30 & 17 & 47 & $\mathbf{0 . 0 4}$
\end{tabular}

The presence of multiple angiographic leaks on FFA was also statistically significantly; observed more in subjects with high stress score levels(19/50; $p$-value=0.03)as shown in Table 4.

Table 4. Effect of stress on number of angiographic leaks

\begin{tabular}{lcccc} 
Level of stress & Single & Multiple & Total & P-value \\
\hline Low & 5 & 0 & 5 & 0.6 \\
\hline Medium & 23 & 5 & 28 & 0.07 \\
\hline High & 31 & 19 & 50 & $\mathbf{0 . 0 3}$
\end{tabular}

The fibrinous sub-retinal fluid was seen in 9 patients $(10.84 \%$; $p$-value $=0.62)$, which was not statistically significant among different grades of stress scores(Table 5), but incidence of this exudation, with poorer outcome,was observed more in severe stress score subjects $(77.77 \% ; 7 / 9)$.

Table 4. Effect of stress on occurrence of fibrinous sub-retinal fluid $(n=9)$

\begin{tabular}{lcc} 
Level of stress Score & Present/Total & P-value \\
\hline Low & $1 / 9$ & 0.90 \\
\hline Medium & $1 / 9$ & 0.79 \\
\hline High & $7 / 9$ & 0.62
\end{tabular}




\section{REFERENCES}

Central serous chorioretinopathy (CSCR) is a common retinal disorder where development of a serous detachment of sensory retina occurs due to a deficient pumping function at the level of retinal pigment epithelium. ${ }^{14-15}$ At present, neither the etiology nor the patho-physiology of CSCR is completely understood due to absence of any published literature on same. A definitive range of psychological and psychophysiological variables have been associated with the increased incidence of CSCR. Conrad et al. pointed out that patients with CSCR were relatively more stressed due to inadequate coping strategies and had significantly higher emotional distress on the Global Severity Index compared to healthy controls. ${ }^{5}$ None of the published literature works has studied the impact of these factors on the clinical profile and varied presentation of acute CSCR patients.

The key findings of our present study are that patients suffering from acute idiopathic CSCR experienced higher psycho-social stress levels. It also pointed out that higher stress levels were associated with more varied presentations of CSCR like bilateral involvement, multiple angiographic leaks and fibrinous sub-retinal fluid. Kim et al also reported that psycho-social stress levels are associated with active stage of disease and correlated to size of choroidal pathology. ${ }^{16}$ Lahousen et al also reported that acute CSCR is related to more unfavourable stress coping and more physical complaints compared to its chronic sub-type. ${ }^{17}$ The atypical presentations such as multifocal leaks with subretinal fibrin can lead to chronic course as well as lesser chances of spontaneous recovery. Therefore, the present study denotes a positive correlation between occurrence of acute CSCR with atypical presentation and higher level of psychosocial stress.

The disease is typically unilateral but over the course of disease, bilateral involvement has been noted in almost $40 \%$ of patients. ${ }^{18}$ On the contrary, our study exhibited a significant association between higher stress levels and bilateral involvement at presentation itself. Acute cases usually present with a single angiographic leak, rarely two or more. ${ }^{19}$ In present study, we also noted out that patients with increased psycho-social stress scores were more likely to have multiple leaks. Fibrin accumulation in the sub-retinal space is relatively uncommon visually damaging complication of severe CSCR with sub-optimal visual recovery. ${ }^{20}$ The incidence of fibrinous sub-retinal fluid was about $10 \%$ in our study with a higher incidence among patients with high stress scores (77.77\%). Thus, higher psycho-social stress levels can present with severe, atypical disease at presentation and lead to suboptimal visual recovery.

The strength of the current study lies in the large number of patients enrolled for studying the effect of different grades of psycho-social stress on clinical profile of CSCR patients. This is perhaps first study providing a better insight on the importance of avoiding stress in the optimal visual recovery in these patients. However, there are inherent limitations in the current study, like Social Readjustment Rating Score used, which may not be ideal for our study population group since different ethnical groups react differently to life events employed to assess stress levels. Another limitation is the lack of age $\&$ sexmatched control group.

In conclusion, higher grade of psycho-social stress can present with more severe forms of acute CSCR with a possible sub-optimal visual recovery. The avoidance of stress, relaxation therapies and stress prevention programs can significantly reduce severe forms of acute CSCR.

\section{CONCLUSION}

The higher grade of psycho-social stress can present with more severe forms of acute CSCR with a possible sub-optimal visual recovery.The avoidance of stress, relaxation therapies and stress prevention programs can significantly reduce severe forms of acute CSCR.

\section{REFERENCES}

1. Spaide RF, Campeas L, Haas A et al. Central serous chorioretinopathy in younger and older adults. Ophthalmology. 1996;103:2070-9.

2. Tittl MK, Spaide RF, Wong D et al. Systemic findings associated with central serous chorioretinopathy. Am J Ophthalmol. 1999;128:63-8.

3. Yannuzzi LA. Type-A behavior and central serous chorioretinopathy. Retina.1987;7:111-31.

4. Tsai DC, Chen SJ, Huang CC et al. Epidemiology of idiopathic central serous chorioretinopathy in Taiwan, 2001-2006: a population-based study. PLoS One.2013;8:66858.

5. Conrad R, Geiser F, Kleiman A et al. Temperament and character personality profile and illness-related stress in 
central serous chorioretinopathy. ScientificWorldJournal.2014;2014:631687.

6. Piskunowicz $M$, Jaracz $M$, Lesiewska $H$ et al. Temperament profile in patients with central serous chorioretinopathy: a case-control study. Eur J Ophthalmol.2014;24:392-5.

7. Piccolino FC, Borgia L. Central serous chorioretinopathy and indocyanine green angiography. Retina.1994;14:23142.

8. Spaide RF, Hall L, Haas A et al. Indocyanine green videoangiography of older patients with central serous chorioretinopathy.Retina.1996;16:203-13.

9. Spahn C, Wiek J, Burger T. Operationalized psychodynamic diagnostics (OPD) in patients with central serous chorioretinopathy. PsychotherPsychosom Med Psychol. 2004; 54:52-7. 25.

10. Conrad R, Bodeewes I, Schilling G, Geiser F, Imbierowicz K, Liedtke R. Central serous chorioretinopathy and psychological stress. Ophthalmology. 2000; 97:527-31.

11. Holmes $\mathrm{TH}$, Rahe RH. The Social Readjustment Rating Scale. Journal of Psychosomatic Research.August 1967; 11:(2),213-218.

12. Sahin A, Bez Y, Kaya MC et al. Psychological distress and poor quality of life in patients with central serous chorioretinopathy. Semin Ophthalmol.2014;29:73-6.

13.Conrad R, Bodeewes I, Schilling G et al. Central serous chorioretinopathy and psychological stress. Ophthalmology. 2000;97:527-31.

14. Wang $M$, Munch IC, Hasler PW, Prünte $C$, Larsen M. Central serous chorioretinopathy.Acta Ophthalmol. 2008;86:126-145.
15. Liu DT, Fok AC, Chan W et al. Central serous chorioretinopathy. In: Ryan SJ, Schachat AP, Wilkinson CP, Hinton DR, Sadda SR, Wiedemann P, editors. Retina. 5th ed.,2012, Elsevier Health Sciences, 1292-1305.

16. Kim YK, WooJ, Park KH, Chi YK, Han JW, Kim KW. Association of Central Serous Chorioretinopathy with Psychosocial Factors is Dependent on Its Phase and Subtype. Korean J Ophthalmol 2018;32(4):281-289.

17. Lahousen $T$, Painold A, Luxenberger W, Schienle A, Kapfhammer HP, Ille R. Psychological factors associated with acute and chronic central serous chorioretinopathy. Nord J Psychiatry. 2016;70(1):24-30.

18. Kitzmann AS, Pulido JS, DiehI NN et al. The incidence of central serous chorioretinopathy in Olmsted County, Minnesota, 1980-2002. Ophthalmology. 2008; 115:169173.

19. Burumcek E, Mudun A, Karacorlu S, Arslan MO. Laser photocoagulation for persistent central serous retinopathy: results of long-term follow-up. Ophthalmology. 1997; 104:616-22.

20. Schatz $H$, McDonald HR et al. Subretinal fibrosis in central serous chorioretinopathy. Ophthalmology. 1995;102:1077-1088.-866.

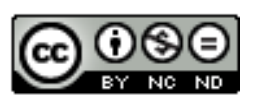

This work licensed under Creative Commons Attribution 